\title{
Neutron Star Interiors and Topology Change
}

\author{
Peter K. F. Kuhfittig \\ Department of Mathematics, Milwaukee School of Engineering, Milwaukee, WI 53202-3109, USA
}

Correspondence should be addressed to Peter K. F. Kuhfittig; kuhfitti@msoe.edu

Received 1 January 2013; Revised 8 March 2013; Accepted 11 March 2013

Academic Editor: Emilio Elizalde

Copyright (C) 2013 Peter K. F. Kuhfittig. This is an open access article distributed under the Creative Commons Attribution License, which permits unrestricted use, distribution, and reproduction in any medium, provided the original work is properly cited.

Quark matter is believed to exist in the center of neutron stars. A combined model consisting of quark matter and ordinary matter is used to show that the extreme conditions existing in the center could result in a topology change, that is, in the formation of wormholes.

\section{Introduction}

Wormholes are handles or tunnels in the spacetime topology connecting different parts of our universe or of different universes. The meticulous analysis in [1] has shown that such wormholes may be actual physical objects permitting twoway passage.

Also introduced in [1] is the metric for a static spherically symmetric wormhole; namely,

$$
d s^{2}=-e^{\phi(r)} d t^{2}+e^{\lambda(r)} d r^{2}+r^{2}\left(d \theta^{2}+\sin ^{2} \theta d \varphi^{2}\right),
$$

where $e^{\lambda(r)}=1 /(1-b(r) / r)$. Here $b=b(r)$ is the shape function and $\phi=\phi(r)$ is the redshift function, which must be everywhere finite to prevent an event horizon. For the shape function we must have $b\left(r_{0}\right)=r_{0}$, where $r=r_{0}$ is the radius of the throat of the wormhole and also $b^{\prime}\left(r_{0}\right)<1$ and $b(r)<r$ to satisfy the flare-out condition [1]. We are using geometrized units where $G=c=1$.

While Morris and Thorne concentrated on traversable wormholes suitable for humanoid travelers (and possible construction by an advanced civilization), a more general question is the possible existence of naturally occurring wormholes [2]. It has also been suggested that the extreme conditions inside a massive compact star may result in a topology change [3]. It is proposed in this paper that a neutron star is a candidate for such a topology change as a result of quark matter [4-7], which is believed to exist at the center of neutron stars [8]. To that end, we will use a two-fluid model consisting of ordinary matter and quark matter based on the MIT bag model [9]. Two cases will be considered: the noninteracting and interacting two-fluid models. (We will concentrate mainly on the former case.) The reason for having two cases is that quark matter is a viable candidate for dark matter [10], albeit under different circumstances, and so is likely to be weakly interacting.

A class of exact solutions of the Einstein-Maxwell field equations describing a wormhole with an anisotropic matter distribution is discussed in [11].

A combined model consisting of neutron-star matter and of a phantom/ghost scalar field yielding a nontrivial topology is discussed in [12]. The model is referred to as a neutron-starplus-wormhole system.

\section{Basic Equations}

For our basic equations we follow [13]. The energy momentum tensor of the two-fluid model is given by

$$
\begin{gathered}
T_{0}^{0} \equiv \rho_{\text {effective }}=\rho+\rho_{q}, \\
T_{1}^{1}=T_{2}^{2} \equiv-p_{\text {effective }}=-\left(p+p_{q}\right) .
\end{gathered}
$$

In (2) and (3), $\rho$ and $p$ correspond to the respective energy density and pressure of the baryonic matter, while $\rho_{q}$ and $p_{q}$ correspond to the energy density and pressure of the quark matter. The left-hand sides are the effective energy density and pressure, respectively, of the composition. 
The Einstein field equations are listed next [13]:

$$
\begin{gathered}
e^{-\lambda}\left(\frac{\lambda^{\prime}}{r}-\frac{1}{r^{2}}\right)+\frac{1}{r^{2}}=8 \pi\left(\rho+\rho_{q}\right), \\
e^{-\lambda}\left(\frac{\phi^{\prime}}{r}+\frac{1}{r^{2}}\right)-\frac{1}{r^{2}}=8 \pi\left(p+p_{q}\right), \\
\frac{e^{-\lambda}}{2}\left[\frac{\left(\phi^{\prime}\right)^{2}-\lambda^{\prime} \phi^{\prime}}{2}+\frac{\phi^{\prime}-\lambda^{\prime}}{r}+\phi^{\prime \prime}\right]=8 \pi\left(p+p_{q}\right),
\end{gathered}
$$

recalling that $T_{1}^{1}=T_{2}^{2}$.

In the MIT bag model, the matter equation of state is given by

$$
p_{q}=\frac{1}{3}\left(\rho_{q}-4 B\right)
$$

where $B$ is the bag constant, which we take to be $145 \mathrm{MeV} /(\mathrm{fm})^{3}[9,14]$. Since quarks are part of the standard model, we assume that $p_{q}>0$; hence $\rho_{q}>4 B$. For normal matter we use the somewhat idealized equation of state [11]

$$
p=m \rho, \quad 0<m<1
$$

Since we are assuming the pressure to be isotropic, the conservation equation is [13]

$$
\frac{d\left(p_{\text {effective }}\right)}{d r}+\frac{1}{2} \phi^{\prime}\left(\rho_{\text {effective }}+p_{\text {effective }}\right)=0
$$

\section{Solutions of Basic Equations}

As noted in the Introduction, we will concentrate mainly on the noninteracting fluid model. This means that the two fluids, normal matter and quark matter, do not interact. The resulting conservation equations are therefore independent of each other. Using (7) and (8), we have

$$
\begin{aligned}
& \frac{d \rho}{d r}+\phi^{\prime}\left(\frac{1+m}{2 m}\right) \rho=0, \\
& \frac{d \rho_{q}}{d r}+2 \phi^{\prime}\left(\rho_{q}-B\right)=0 .
\end{aligned}
$$

The solutions are

$$
\begin{gathered}
\rho=\rho_{0} e^{-\phi(1+m) / 2 m}, \\
\rho_{q}=B+\rho_{(q, 0)} e^{-2 \phi}
\end{gathered}
$$

where $\rho_{0}$ and $\rho_{(q, 0)}$ are integration constants. Equations (4), (5), and (6) now give

$$
\begin{array}{r}
e^{-\lambda}\left[-\frac{\lambda^{\prime} \phi^{\prime}}{2}+\frac{\left(\phi^{\prime}\right)^{2}}{2}+\phi^{\prime \prime}+\frac{2 \phi^{\prime}}{r}\right] \\
=8 \pi\left[\rho(1+3 m)+\left(2 \rho_{q}-4 B\right)\right] .
\end{array}
$$

It becomes apparent that this equation is linear in $e^{-\lambda}$ once it is rewritten in the following form:

$$
\begin{aligned}
\left(e^{-\lambda}\right)^{\prime}+ & e^{-\lambda}\left(\phi^{\prime}+\frac{2 \phi^{\prime \prime}}{\phi^{\prime}}+\frac{4}{r}\right) \\
& =\frac{2}{\phi^{\prime}}(8 \pi)\left[\rho(1+3 m)+\left(2 \rho_{q}-4 B\right)\right] .
\end{aligned}
$$

The integrating factor is

$$
\text { I.F. }=e^{\phi+2 \ln \phi^{\prime}+4 \ln r}=e^{\phi}\left(\phi^{\prime}\right)^{2} r^{4},
$$

leading at once to

$$
\begin{aligned}
& \frac{d}{d r}\left[e^{-\lambda} e^{\phi}\left(\phi^{\prime}\right)^{2} r^{4}\right] \\
& \quad=e^{\phi}\left(\phi^{\prime}\right)^{2} r^{4}\left(\frac{2}{\phi^{\prime}}\right)(8 \pi)\left[\rho(1+3 m)+\left(2 \rho_{q}-4 B\right)\right] .
\end{aligned}
$$

To write the solution for $e^{-\lambda}$, we first define $F(r)=\rho(1+3 m)+$ $\left(2 \rho_{q}-4 B\right)$, which becomes, after substituting the solutions $\rho$ and $\rho_{q}$,

$$
F(r)=\rho_{0} e^{-\phi(1+m) / 2 m}(1+3 m)+2 \rho_{(q, 0)} e^{-2 \phi}-2 B .
$$

We now have

$$
e^{-\lambda}=\frac{16 \pi}{e^{\phi}\left(\phi^{\prime}\right)^{2} r^{4}} \int_{r_{0}}^{r} e^{\phi} \phi^{\prime} r_{1}^{4} F\left(r_{1}\right) d r_{1} .
$$

The lower limit $r=r_{0}$ is the radius of the throat of the wormhole, as we will see.

As noted in Section 1, the shape function $b=b(r)$ is obtained from $\mathrm{e}^{-\lambda(r)}$, so that

$$
b(r)=r\left(1-e^{-\lambda(r)}\right) .
$$

We obtain

$$
b(r)=r\left[1-\frac{16 \pi}{e^{\phi}\left(\phi^{\prime}\right)^{2} r^{4}} \int_{r_{0}}^{r} e^{\phi} \phi^{\prime} r_{1}^{4} F\left(r_{1}\right) d r_{1}\right]
$$

Note especially that $b\left(r_{0}\right)=r_{0}$, which characterizes the throat of the wormhole.

To study the other requirement, $0<b^{\prime}\left(r_{0}\right)<1$, we start with

$$
b^{\prime}(r)=1-e^{-\lambda(r)}+r\left[-\frac{d}{d r} e^{-\lambda(r)}\right],
$$

which leads to

$$
\begin{aligned}
b^{\prime}\left(r_{0}\right)=1+r_{0} \frac{16 \pi}{\phi^{\prime}\left(r_{0}\right)}[ & -\rho_{0} e^{-\phi\left(r_{0}\right)(1+m) / 2 m}(1+3 m) \\
& \left.-2 \rho_{(q, 0)} e^{-2 \phi\left(r_{0}\right)}+2 B\right] .
\end{aligned}
$$


Given that $\phi^{\prime}$, the gradient of the redshift function, is related to the tidal force, one would expect its magnitude to be extremely large. So $b^{\prime}\left(r_{0}\right)$ will indeed be less than unity if the expression inside the brackets in (22) is negative and sufficiently small in absolute value. If this is the case, then the flare-out condition is met; that is,

$$
\frac{r_{0} b^{\prime}\left(r_{0}\right)-b\left(r_{0}\right)}{2\left[b\left(r_{0}\right)\right]^{2}}<0
$$

Since it indicates a violation of the weak energy condition, the flare-out condition is the primary prerequisite for the existence of wormholes [1]. (The condition $b(r) / r<1$ will be checked later.)

Unfortunately, the constants $B$ and $\rho_{(q, 0)}$ are also large, so that (22) must be examined more closely.

\section{The Gradient of the Redshift Function}

To analyze (22), we start with the gradient of $\phi[15]$ :

$$
\frac{d \phi}{d r}=\left[\frac{2 G m(r)}{c^{2} r^{2}}+8 \pi r \frac{G}{c^{4}} p\right] \frac{1}{1-2 G m(r) / c^{2} r},
$$

where $m(r)$ is the total mass-energy inside the radial distance $r$. (This formula is also used in the derivation of the TolmanOppenheimer-Volkoff equation.) In geometrized units we get, for $r=r_{0}$,

$$
\phi^{\prime}\left(r_{0}\right)=\frac{2 m\left(r_{0}\right) / r_{0}^{2}+8 \pi r_{0} p\left(r_{0}\right)}{1-2 m\left(r_{0}\right) / r_{0}} .
$$

Since we are now concerned with what is going to become the throat at $r=r_{0}$, we need to evaluate $\rho_{0}$ and $\rho_{(q, 0)}$ in (11) and (12) to obtain

$$
\begin{gathered}
\rho_{0}=\rho\left(r_{0}\right) e^{\phi\left(r_{0}\right)(1+m) / 2 m}, \\
\rho_{(q, 0)}=e^{2 \phi\left(r_{0}\right)}\left[\rho_{q}\left(r_{0}\right)-B\right] .
\end{gathered}
$$

So (22) becomes

$$
b^{\prime}\left(r_{0}\right)=1+\frac{16 \pi r_{0}\left[-\rho\left(r_{0}\right)(1+3 m)-2 \rho_{q}\left(r_{0}\right)+4 B\right]}{\left[2 m\left(r_{0}\right) / r_{0}^{2}+8 \pi r_{0} p\left(r_{0}\right)\right] /\left[1-2 m\left(r_{0}\right) / r_{0}\right]} .
$$

We already know that $p=m \rho$ and that $\rho_{q}>4 B$, so that the numerator is indeed negative. For the purpose of comparison, we will simply assume for now that $\rho_{q}\left(r_{0}\right)=4 B$. Then

$$
b^{\prime}\left(r_{0}\right)=1+\frac{16 \pi\left[-\rho\left(r_{0}\right)(1+3 m)-4 B\right]}{\left[2 m\left(r_{0}\right) / r_{0}^{3}+8 \pi p\left(r_{0}\right)\right] /\left[1-2 m\left(r_{0}\right) / r_{0}\right]} .
$$

Disregarding $r_{0}$ for the time being, suppose that we determine $m\left(r_{0}\right)$ so that $2 m\left(r_{0}\right)$ exceeds $16 \pi(4 B)$. We obtain

$$
16 \pi(4 B)=16 \pi(4)(145) \frac{\mathrm{MeV}}{(\mathrm{fm})^{3}} \frac{G}{c^{4}} \mathrm{~m}^{-2},
$$

which leads to $m\left(r_{0}\right) \approx 10^{-8} \mathrm{~m}$. The other term in the denominator, $8 \pi p\left(r_{0}\right)$, could be close to zero (since $m>0$ ). However, by simply doubling the value of $m\left(r_{0}\right)$, we can compensate for the normal-matter term $-\rho\left(r_{0}\right)(1+3 m)$ in the numerator, since this term is smaller than the other term. Given that the density of nuclear matter ranges from $6 \times 10^{17} \mathrm{~kg} / \mathrm{m}^{3}$ to $8 \times$ $10^{17} \mathrm{~kg} / \mathrm{m}^{3}$, we may take $\rho_{q}$ to be about $2 \times 10^{18} \mathrm{~kg} / \mathrm{m}^{3}$. So in geometrized units, $\rho_{q}\left(r_{0}\right)$ is in fact larger than and reasonably close to $4 B$, thereby justifying the assumption $\rho_{q}\left(r_{0}\right) \approx 4 B$.

Remark 1. The assumption $\rho_{q}\left(r_{0}\right) \approx 4 B$ yields $-2 \rho_{q}\left(r_{0}\right)+4 B \approx$ $-4 B$, leading to a negative numerator in (28). It is conceivable that the bag pressure $B$ increases with increasing density, but to reverse the sign, the value would have to be more than doubled for a fixed $\rho_{q}$. More importantly, however, (7) suggests that this increase is likely to be compensated for by a concomitant increase in $\rho_{q}$.

Next, given the enormous density near the center of a neutron star, $m\left(r_{0}\right) \approx 10^{-8} \mathrm{~m}$ would correspond to a fairly small value for $r_{0}$ : using $\rho_{q}\left(r_{0}\right) \approx 4 B$ again,

$$
\frac{4}{3} \pi r_{0}^{3} \rho_{q}\left(r_{0}\right)=10^{-8}
$$

yields $r_{0} \approx 1 \mathrm{~m}$, although larger values would be allowed. So the above estimate holds.

Finally, observe that the other factor in the denominator, $1-2 m\left(r_{0}\right) / r_{0}$, is now seen to be very close to unity.

We conclude that $b^{\prime}\left(r_{0}\right)<1$. So in the neighborhood of the throat, $b(r) / r<1$. To show that this inequality holds away from the throat, recall that $(1 / 2) b(r)$ is the total mass inside a sphere of radius $r$. Hence

$$
\begin{gathered}
\frac{1}{2} b(r)=\int_{r_{0}}^{r} 4 \pi r^{2} \rho(r) d r, \\
\frac{1}{2} b^{\prime}(r)=4 \pi r^{2} \rho(r) .
\end{gathered}
$$

Since $\rho(r)$ decreases as $r$ increases, $b^{\prime}(r)$ is positive but decreasing, so that $b(r) / r$ remains less than unity for $r>r_{0}$.

\section{Completing the Wormhole Structure}

As we have seen, $m\left(r_{0}\right)$ is the mass inside the radial distance $r=r_{0}$. Since $r=r_{0}$ is now the throat of the wormhole, it follows from the definition of throat that the interior $r<r_{0}$ is outside the manifold. Although not part of the wormhole spacetime, it still contributes to the gravitational field. This can be compared to a thin-shell wormhole from a Schwarzschild black hole [16]: while not part of the manifold, the black hole generates the gravitational field. This comparison explains why our wormhole requires a minimum throat radius to help produce a sufficiently strong gravitational field.

Since the throat of the wormhole is deep inside the neutron star, it cannot be directly observed. According to [12], however, there may still be observable tell-tale signs: if two neutron stars are connected by a wormhole, they would have identical, or nearly identical, characteristics. An even 
stronger indication would be any observed variation in the mass of a neutron star.

Remark 2. The quark-matter core, being surrounded by nuclear matter, raises a question regarding the interface region. As noted in the Introduction, quark matter is believed to be weakly interacting. So the most likely result is a drop in the energy density in the neighborhood of the core's surface.

5.1. The Need for Quark Matter. If quark matter is eliminated from the model, then the right-hand side of (13) becomes $8 \pi \rho(1+3 m)$. As a consequence, the $4 B$ term in (28) is also eliminated. Since $m>0$ and $16>8 / 3$,

$$
\left|16 \pi\left[-\rho\left(r_{0}\right)(1+3 m)\right]\right|>2 \times \frac{4}{3} \pi \rho\left(r_{0}\right)=\frac{2 m\left(r_{0}\right)}{r_{0}^{3}} .
$$

So the fraction in (28) exceeds in absolute value the following:

$$
\frac{-\left[2 m\left(r_{0}\right) / r_{0}^{3}\right](1+3 m)}{\left[2 m\left(r_{0}\right) / r_{0}^{3}+8 \pi m \rho\left(r_{0}\right)\right] /\left[1-2 m\left(r_{0}\right) / r_{0}\right]}
$$

Although we are now dealing with nuclear matter $\left(\rho \approx 10^{-10}\right)$, we still have $1-2 m\left(r_{0}\right) / r_{0} \approx 1$. It is quite evident that for $m$ close to zero, the fraction in statement (33) is slightly larger than unity in absolute value; hence $b^{\prime}\left(r_{0}\right)$ is negative, which is absurd. As $m$ increases, $b^{\prime}\left(r_{0}\right)$ only decreases further, so that the flare-out condition cannot be met.

It now becomes apparent that our conclusion depends critically on the fact that $B$ is a constant: increase the throat size until $2 m\left(r_{0}\right)$ surpasses $16 \pi(4 B)$. So based on our model, a topology change is possible only if there is a sufficient amount of quark matter in the center. More precisely, the assumption $\rho\left(r_{0}\right) \approx 4 B$ implies that the quark matter has to extend at least to $r=r_{0}$.

5.2. The Interacting Case. We conclude with some brief comments on the interacting case. If the two fluids are assumed to interact, then the conservation equations take on the following forms $[13,17]$ :

$$
\begin{gathered}
\frac{d \rho}{d r}+\phi^{\prime}\left(\frac{1+m}{2 m}\right) \rho=Q, \\
\frac{d \rho_{q}}{d r}+2 \phi^{\prime}\left(\rho_{q}-B\right)=-3 Q .
\end{gathered}
$$

The quantity $Q$ expresses the interaction between the two types of matter and falls off rapidly as $r \rightarrow \infty$. Furthermore, since the interaction is assumed to be relatively weak, $Q$ is a very small quantity compared to $B$. So in comparing $m\left(r_{0}\right)$ and $\rho_{q}\left(r_{0}\right)$ in the expression for $b^{\prime}\left(r_{0}\right)$, the presence of the quantity $Q$ is going to have little effect.

\section{Conclusion}

Quark matter is believed to exist in the center of neutron stars. The analysis in this paper is therefore based on a two-fluid model comprising ordinary and quark matter with an isotropic matter distribution. It is shown that the extreme conditions may result in a topology change; that is, for certain choices of the matter content in the Einstein field equations, a neutron star could give rise to a wormhole.

\section{References}

[1] M. S. Morris and K. S. Thorne, "Wormholes in spacetime and their use for interstellar travel: a tool for teaching general relativity", American Journal of Physics, vol. 56, no. 5, pp. 395412, 1988.

[2] P. K. F. Kuhfittig, "Could some black holes have evolved from wormholes?” Scholarly Research Exchange, vol. 2008, Article ID 296158, 5 pages, 2008.

[3] A. DeBenedictis, R. Garattini, and F. S. N. Lobo, "Phantom stars and topology change," Physical Review D, vol. 78, no. 10, Article ID 104003, 14 pages, 2008.

[4] N. Itoh, "Hydrostatic equilibrium of hypothetical quark stars," Progress of Theoretical Physics, vol. 44, no. 1, pp. 291-292, 1970.

[5] U. Heinz and M. Jacob, "Evidence for a new state of matter: an assessment of the results from the CERN lead beam programme," http://arxiv.org/abs/nucl-th/0002042.

[6] T. Schaefer, "Quark matter," http://arxiv.org/abs/hep-ph/ 0304281.

[7] B. Müller, "Quark matter 2005-theoretical summary," http:// arxiv.org/abs/nucl-th/0508062.

[8] M. A. Perez-Garcia, J. Silk, and J. R. Stone, "Dark matter, neutron stars, and strange quark matter," Physical Review Letters, vol. 105, no. 14, Article ID 141101, 4 pages, 2010.

[9] A. Chodos, R. L. Jaffe, K. Johnson, C. B. Thorn, and V. F. Weisskopf, "New extended model of hadrons," Physical Review D, vol. 9, no. 12, pp. 3471-3495, 1974.

[10] A. Bhattacharyya, J. E. Alam, S. Sarkar et al., "Cosmological QCD phase transition and dark matter," Nuclear Physics A, vol. 661, no. 1-4, pp. 629-632, 1999.

[11] F. Rahaman, M. Kalam, and K. A. Rahman, "Wormhole geometry from real feasible matter sources," International Journal of Theoretical Physics, vol. 48, no. 2, pp. 471-475, 2009.

[12] V. Dzhunushaliev, V. Folomeev, B. Kleihaus, and J. Kunz, "Mixed neutron-star-plus-wormhole systems: equilibrium configurations," Physical Review D, vol. 85, no. 12, Article ID 124028, 14 pages, 2012.

[13] R. Rahaman, P. K. F. Kuhfittig, R. Amin, G. Mandal, S. Ray, and N. Islam, "Quark matter as dark matter in modeling galactic halo," Physics Letters B, vol. 714, no. 2-5, pp. 131-135, 2012.

[14] F. Rahaman, R. Maulick, R. Sharma, S. Ray, and I. Karar, "Strange stars in Krori-Barua space-time," http://arxiv.org/ abs/1108.6125.

[15] F. Weber, R. Negreiros, and P. Rosenfield, "Neutron star interiors and the equation of state of superdense matter," http://arxiv.org/abs/0705.2708.

[16] M. Visser, "Traversable wormholes from surgically modified Schwarzschild spacetimes," Nuclear Physics B, vol. 328, no. 1, pp. 203-212, 1989.

[17] D. Pavón and B. Wang, "Le Châtelier-Braun principle in cosmological physics," General Relativity and Gravitation, vol. 41, no. 1, pp. 1-5, 2009. 


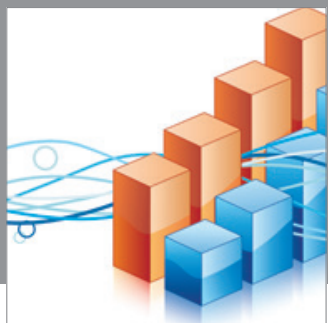

Advances in

Operations Research

mansans

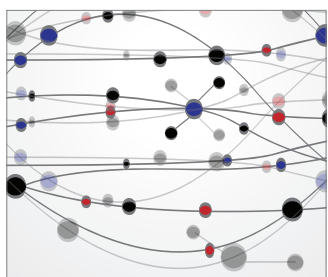

The Scientific World Journal
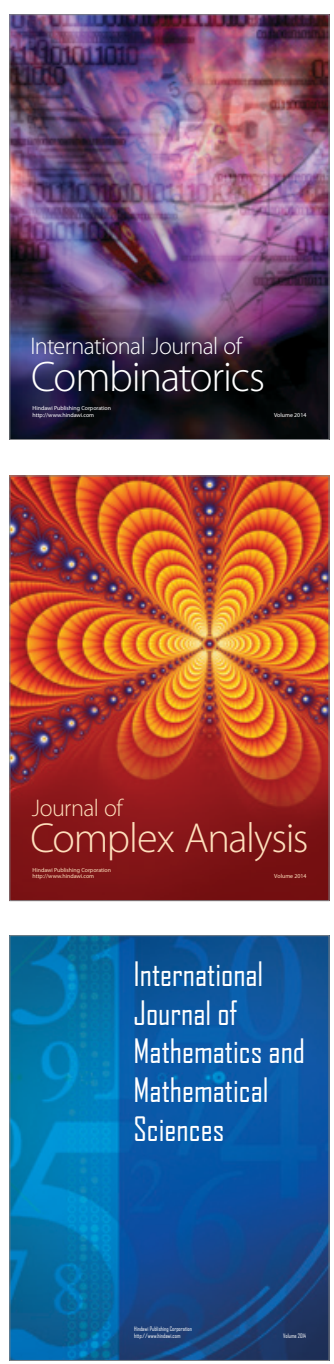
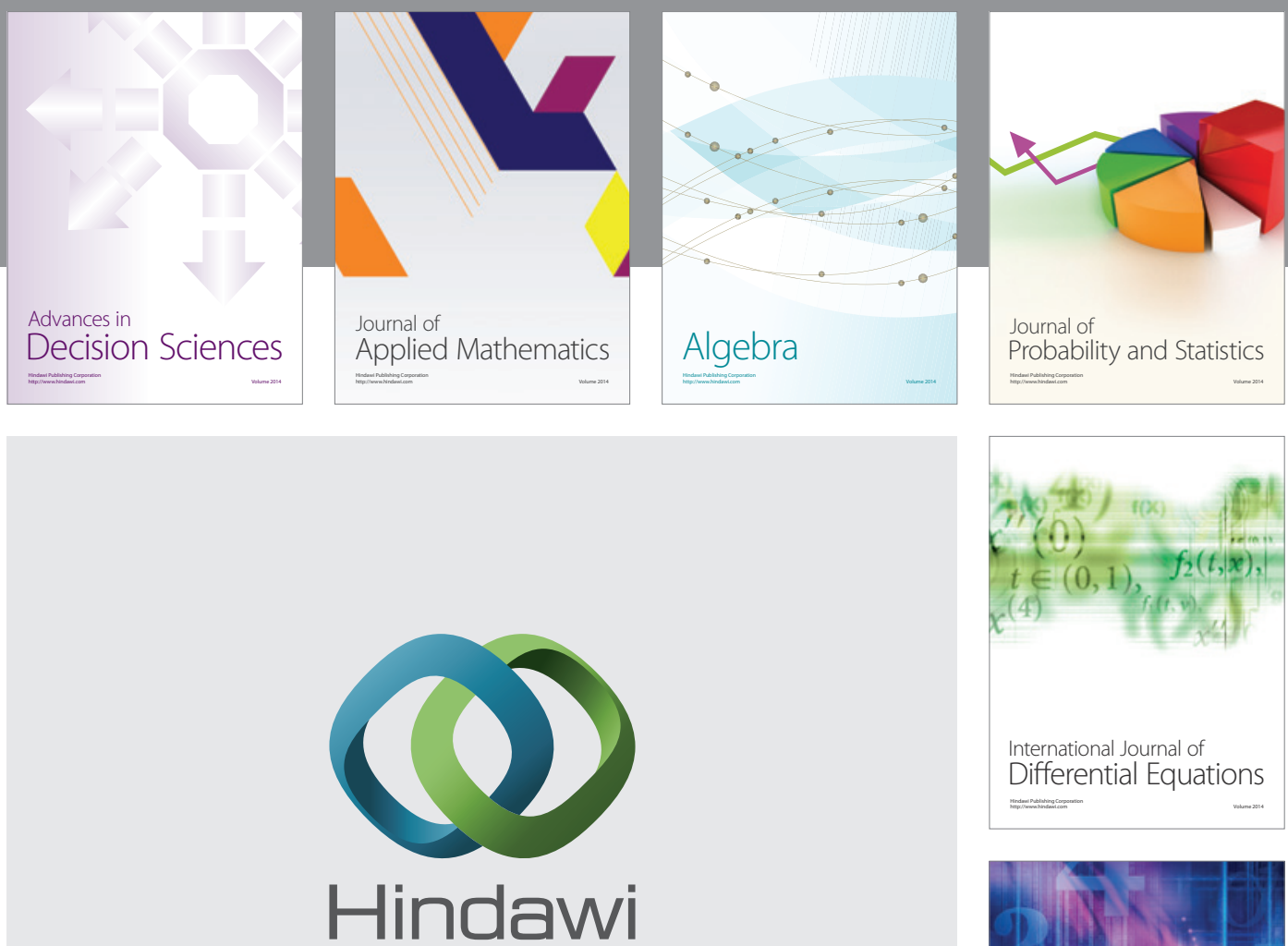

Submit your manuscripts at http://www.hindawi.com
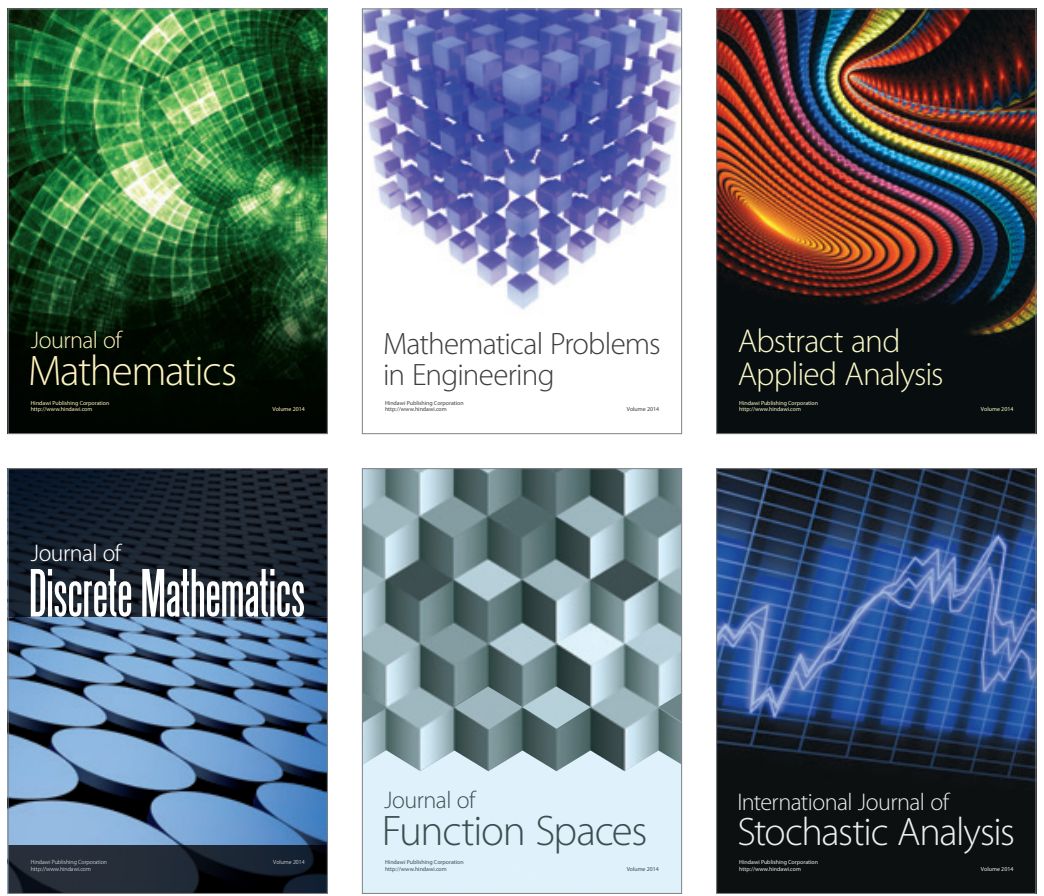

Journal of

Function Spaces

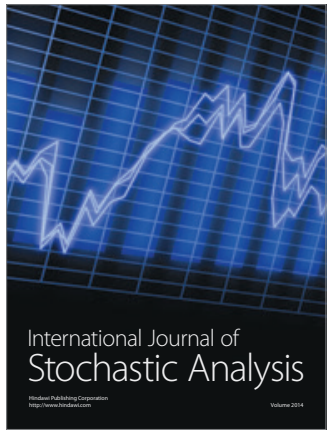

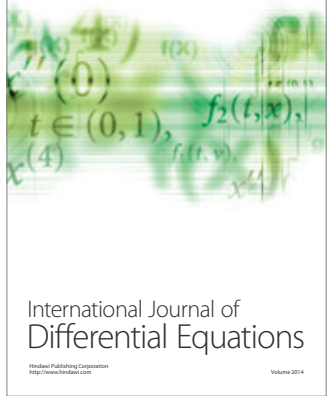
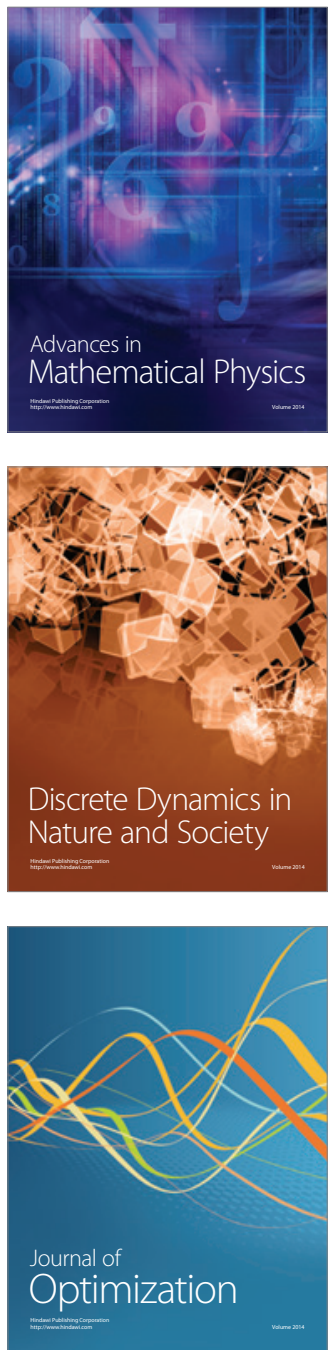